\title{
Model of collective decision-making in nestmate recognition fails to account for individual discriminator responses and non-independent discriminator errors
}

\author{
Margaret J. Couvillon • Jelle S. van Zweden • Francis L. W. Ratnieks
}

Received: 13 September 2011 / Revised: 13 October 2011 /Accepted: 29 November 2011 /Published online: 22 December 2011

(C) Springer-Verlag 2011

In their paper "Nestmate recognition in social insects: overcoming physiological constraints with collective decision making", Johnson and co-authors present agent-based models of collective decision-making to show that multiple discriminators (guards) may together mitigate individual decision errors and achieve near-perfect, colony-level discrimination of nestmates from non-nestmates (Johnson et al. 2011). The authors also conduct a literature review of discrimination errors in social insects to evaluate, in contrast to their model, the theory of optimal acceptance thresholds, which predicts that falsely rejecting nestmates and correctly rejecting non-nestmates should be negatively correlated. They conclude that as false rejections are exceedingly rare across species, there can be no correlation between the two errors. However, we believe that the conclusions drawn from both the model and the literature review are based on false assumptions, which we will detail later.

The models put forward by the authors test the effect of guard density and guard-intruder encounter rate on the acceptance levels of nestmates and non-nestmates. Overall, they demonstrate that the probability of rejection increases with increasing encounters. For example, in their first mod$\mathrm{el}$, the overall probability of acceptance falls to less than $1 \%$

Communicated by J. Traniello and M. Beekman

M. J. Couvillon $(\bowtie) \cdot$ J. S. van Zweden · F. L. W. Ratnieks

The Laboratory of Apiculture and Social Insects (LASI),

School of Life Sciences, The University of Sussex,

Falmer, Brighton BN1 9QG, UK

e-mail: M.Couvillon@Sussex.ac.uk

J. S. van Zweden

Centre for Social Evolution, Department of Biology,

University of Copenhagen,

Universitetsparken 15,

2100 Copenhagen Ø, Denmark after only three encounters at an $80 \%$ per-encounter rejection probability, but this also occurs after 21 encounters at $20 \%$ per-encounter rejection probability. Given that the model's results show that increasing the discriminator interactions increases the probability of rejection, the authors suggest that recruitment of additional guards may be an alternative to the optimal acceptance threshold theory, whereby guards exhibit an individual, context-dependent response to incoming intruders that is based on the frequency of intruder contact and the cost of making errors (Reeve 1989; Starks et al. 1998).

In our opinion, their hypothesis and models are intriguing to consider and could in principle work. The idea that colonies up-regulate the number of guards at the entrance when frequency of robbing increases has already been both observed in a natural situation and tested experimentally (Downs and Ratnieks 2000; Couvillon et al. 2008, 2009). When nectar supplies dwindle (for example, as we move from summer into autumn), honeybees are more likely to intrude into neighbouring colonies to rob them of stored honey, to which colonies respond over several weeks/ months by stationing more guards at the nest entrance (Downs and Ratnieks 2000; Couvillon et al. 2009). This up-regulation of guard number is also present over shorter time scales: when robbing is experimentally induced, honeybee colonies respond in $15 \mathrm{~min}$ by increasing the number of guards by $20 \%$ (Couvillon et al. 2008). These data initially appear to support the authors' model of collective decision-making.

However, we believe that a few issues have been overlooked. One major problem is that, despite fitting their final model to the biology of the honeybee (Apis mellifera), the authors do not consider previous empirical work investigating individual-level responses of guards to increased intrusion. In addition to demonstrating the rapid up-regulation in 
guard number in response to robbing, Couvillon et al. (2008) also demonstrated that individual guards respond to the increase in intrusion: a guard becomes significantly less accepting, rejecting $28 \%$ and $24 \%$ more nestmates and nonnestmates, respectively. This individual decrease in acceptance is nearly identical to the observed colony-level decrease in acceptance, where guards collectively reject $22 \%$ more nestmates and 29\% more non-nestmates. This difference between the change in individual acceptance and the change in colony-level acceptance is highly non-significant $(p=0.95)$, indicating that individual responses may completely account for the colony-level response. Secondly, Couvillon et al. (2008) also monitored the number of natural fights per individual guard on the entrance platform, which may serve as a proxy for an individual guard's (non-) permissiveness. Overall, there was a rapid, 10-fold increase in the number of natural fights on the entrance platform when robbing was experimentally induced. This response at the individual level far outweighs the modest $20 \%$ colony-level increase of guard numbers. Taken together, the shift in individual acceptance threshold and the increase in number of natural fights strongly suggest that the main component of the increased overall rejection of incomers was the less permissive guarding of each individual guard and not simply because there were more guards (Couvillon et al. 2008). Similar individual responses were seen in wasps (Starks et al. 1998) and ants (Fürst et al. 2011).

A second issue is that the model is built on the idea that errors of one guard are completely independent of the errors of another guard. If this were true, then yes, it would be impossible for an intruder to get by multiple guards. However, we do not believe that this is the case, as discriminating individuals of many social insects derive their recognition template and body odours, which are used for discrimination, from their nestmates and nesting materials (e.g. comb wax in honeybees or coating resin in stingless bees) (D'Ettorre et al. 2006; Couvillon and Ratnieks 2008; van Zweden et al. 2010; van Zweden and d'Ettorre 2010). Therefore, guards usually possess approximately the same recognition template (Breed and Bennett 1987; Breed et al. 2004; Couvillon et al. 2007), and the errors of one guard are thus likely to be similar to the errors of the next guard, so that there will be a significant proportion of intruders that have either a $0 \%$ or $100 \%$ per-encounter rejection probability. We also note that the empirical result of Downs and Ratnieks (2000) showing $20 \%$ of nestmates rejected by the first guard does not necessarily mean that each nestmate has a $20 \%$ per-encounter rejection probability. There is a small but significant difference between these two concepts. It is more likely that rejection follows a probability function that depends on the guards' olfactory acuity and is normally distributed around a particular acceptance threshold; an intruder's odour profile determines her rejection rate, but some intruders therefore have a $0 \%$ or $100 \%$ per-encounter rejection chance. In support of the non-independency of guard errors, Couvillon et al. (2008) found the similar rejection probability for the first guard as when multiple guards had interacted with the intruder. Therefore, having multiple guards could still fail to achieve near-perfect recognition, and it is unrealistic to assume that guards act independently when they are all using the same or similar colony odour template.

Our last point is in regard to the literature review, in which the authors find that "the probability of correct (true) rejection of non-nestmates is quite variable" and "nearly all the studies show no false rejections [of nestmates]" (Johnson et al. 2011). They therefore conclude that there could be no correlation between true and false rejections, which argues against an adaptive acceptance threshold. We have some concerns. Firstly, the correlation between true rejection of non-nestmates and false rejections of nestmates only is predicted within, not between, species (Reeve 1989; Sherman et al. 1997; Couvillon et al. 2009). The recognition cue distributions and sensory systems differ extensively with different organisms; no correlation in error is expected. Therefore, the meta-analysis conducted by the authors has little value to the issue. Secondly, many of the cited studies were not carried out on dedicated guards at the nest entrance: nestmate recognition is context-dependent (Starks et al. 1998), meaning that true guards act aggressively and discriminate when they sense they are at their nest entrance. Their behaviours change to become more permissive when they are not at the nest entrance, and hence the level of false rejection of nestmates may be underestimated in some of the studies. Lastly, there are many more published data of false nestmate rejection rates in honeybees, ranging from 17 to $33 \%$, than listed in the Johnson et al. review of empirical data (Downs et al. 2000; D'Ettorre et al. 2006; Couvillon et al. 2008, 2009; Jones et al. 2011). Most importantly, the appreciable levels of false non-nestmate acceptances (Johnson et al. 2011) are exactly the opposite pattern of what we would expect if the Johnson et al. model were true: the model demonstrates that an increasing number of discriminating individuals should cause the number of rejections to increase quickly for both non-nestmates and nestmates. This discrepancy between model and empirical data leaves us unsure about the applicability of the model.

All in all, the hypothesis posed by Johnson et al. (2011) could benefit from a thorough test, where guards are individually marked and intruders are introduced multiple times, so that the per-guard contact and rejection rates and the nonindependency of their errors can be calculated. If the relationship between rejection rates and guard numbers found can be explained solely by the effect described in the Johnson et al. model, this would be evidence against the theory of individual 
optimal acceptance thresholds. Alternatively, it may be that recognition is more than just the number of guards or encounters. If the latter is the case, it would confirm what was previously shown, where individual acceptance rates do not significantly differ from overall acceptance rates (Couvillon et al. 2008) and would provide additional support for the acceptance threshold model (Reeve 1989; Starks et al. 1998; Downs and Ratnieks 2000; Hauber et al. 2006; Fürst et al. 2011; d'Ettorre et al. 2004).

\section{References}

Breed MD, Bennett B (1987) Kin recognition in highly eusocial insects. In: Fletcher DJC, Michener CD (eds) Kin recognition in animals. John Wiley, Chichester, pp 243-285

Breed MD, Perry S, Bjostad LB (2004) Testing the blank slate hypothesis: why honey bee colonies accept young bees. Insect Soc 51 (1):12-16

Couvillon MJ, Ratnieks FLW (2008) Odour transfer in stingless bee marmelada (Frieseomelitta varia) demonstrates that entrance guards use an "undesirable-absent" recognition system. Behav Ecol Sociobiol 62(7):1099-1105

Couvillon MJ, Caple JP, Endsor SL, Kärcher M, Russell TE, Storey DE, Ratnieks FLW (2007) Nest-mate recognition template of guard honeybees (Apis mellifera) is modified by wax comb transfer. Biol Letters 3(3):228-230

Couvillon MJ, Robinson EJH, Atkinson B, Child L, Dent KR, Ratnieks FLW (2008) En Garde: rapid shifts in honey bee (Apis mellifera) guarding behavior is triggered by onslaught of conspecific intruders. Anim Behav 76:225-232

Couvillon MJ, Roy GGF, Ratnieks FLW (2009) Recognition errors by honey bee (Apis mellifera) guards demonstrate overlapping cues in conspecific recognition. J Apicult Res 48:225-232

d'Ettorre P, Brunner E, Wenseleers T, Heinze J (2004) Knowing your enemies: seasonal dynamics of host-social parasite recognition. Naturwissenschaften 91(12):594-597
D'Ettorre P, Wenseleers T, Dawson J, Hutchinson S, Boswell T, Ratnieks FLW (2006) Wax combs mediate nestmate recognition by guard honeybees. Anim Behav 71:773-779

Downs SG, Ratnieks FLW (2000) Adaptive shifts in honey bee (Apis mellifera $L$.) guarding behavior support predictions of the acceptance threshold model. Behav Ecol 11(3):326-333

Downs SG, Ratnieks FLW, Jefferies SL, Rigby HE (2000) The role of floral oils in the nestmate recognition system of honey bees (Apis mellifera L.). Apidologie 31(3):357-365

Fürst MA, Durey M, Nash DR (2011) Testing the adjustable threshold model for intruder recognition on Myrmica ants in the context of a social parasite. Proc R Soc B. doi:10.1098/rspb.2011.0581

Hauber ME, Moskát C, Bán M (2006) Experimental shift in hosts' acceptance threshold of inaccurate-mimic brood parasite eggs. Biol Lett 2 (2):177-180. doi:10.1098/rsbl.2005.0438

Johnson B, van Wilgenburg E, Tsutsui N (2011) Nestmate recognition in social insects: overcoming physiological constraints with collective decision making. Behav Ecol Sociobiol 65 (5):935-944

Jones S, van Zweden J, Grüter C, Menezes C, Alves D, Nunes-Silva P, Czaczkes T, Imperatriz-Fonseca V, Ratnieks F (2011) The role of wax and resin in the nestmate recognition system of a stingless bee, Tetragonisca angustula. Behav Ecol Sociobiol:1-12. doi:10.1007/s00265-011-1246-7

Reeve HK (1989) The evolution of conspecific acceptance thresholds. Am Nat 133(3):407-435

Sherman PW, Reeve HK, Pfennig DW (1997) Recognition systems. In: Krebs JR, Davies NB (eds) Behavioural ecology. Blackwell, Oxford, pp 69-96

Starks PT, Fischer DJ, Watson RE, Melikian GL, Nath SD (1998) Context-dependent nestmate-discrimination in the paper wasp, Polistes dominulus: a critical test of the optimal acceptance threshold model. Anim Behav 56:449-458

van Zweden JS, d'Ettorre P (2010) Nestmate recognition in social insects and the role of hydrocarbons. In: Blomquist GJ, Bagnères A-G (eds) Insect hydrocarbons: biology, biochemistry, and chemical ecology. Cambridge University Press, Cambridge, pp 222-243

van Zweden JS, Brask JB, Christensen JH, Boomsma JJ, Linksvayer TA, d'Ettorre P (2010) Blending of heritable recognition cues among ant nestmates creates distinct colony gestalt odours but prevents withincolony nepotism. J Evolution Biol 23(7):1498-1508 